\title{
Modelling and Control of a Gravity Gradient Stabilised Satellite
}

\author{
AAGE SKULLESTAD $\dagger$ \\ Keywords: 3-axes stabilisation, satellite, multivariable control, mathematical \\ model \\ This paper describes attitude control, i.e., 3-axes stabilisation and pointing, of a \\ proposed Norwegian small gravity gradient stabilized satellite to be launched into \\ low earth orbit. Generally, a gravity gradient stabilised system has limited stability \\ and pointing capabilities, and wheels and/or magnetic coils are added in order to \\ improve the attitude control. The best attitude accuracy is achieved using wheels, \\ which can give accuracies down to less than one degree, but wheels increase the \\ complexity and cost of the satellite. Magnetic coils allow cheaper satellites, and are \\ an attractive solution to small, inexpensive satellites in low earth orbits and may \\ provide an attitude control accuracy of a few degrees. Scientific measurements often \\ require accurate attitude control in one or two axes only. Combining wheel and coil \\ control may, in these cases, provide the best solutions. The simulation results are \\ based on a linearised mathematical model of the satellite.
}

\section{Introduction}

Kongsberg Defence \& Aerospace has participated in an industrial group concerned with the development of a small Norwegian satellite. The satellite is intended to flow in a low orbit at 800-1200 km altitude and the mission is to do scientific measurements. Earth environment monitoring, which may require a slightly larger satellite, have also been considered. The satellite is expected to be box-shaped, have a total mass of approximately $80 \mathrm{~kg}$ and be gravity gradient stabilised. A proposed $6 \mathrm{~m}$ long gravity boom, including a tip mass of $2 \mathrm{~kg}$, will play a key role in the gravity gradient stabilisation. The gravity forces acting on the satellite are small, and magnetic coil or wheel control is added to improve the attitude control. The satellite is to be three-axis stabilised with its boom pointing outwards. The satellite is illustrated in Fig. 1.

The satellite will during separation from the launch vehicle be exposed to large forces, and tumbling may occur. A detumbling mode is activated in order to calm down the movements. The gravitation boom will be deployed first when the movement of the satellite is sufficiently small. This paper deals with attitude control after the detumbling mode has successfully ended and the boom is fully deployed. At this time all angles are assumed to be small and a linearised mathematical model of the satellite can be justified and is also applied in this paper. All simulations are performed using MATLAB.

Gravity gradient stabilisation has been used in attitude control since the early sixties (Hughcs 1986), but accurate three-axis control has not been achieved using gravity gradient stabilisation alone. Gravity gradient stabilisation combined with magnetic torquing, has gained increased attention as an attractive attitude control system (ACS) for small cheap satellites and is also proposed used in this satellite. Magnetic coils, mounted in the $\mathrm{x}, \mathrm{y}$ and $\mathrm{z}$ facets of the satellite main body, perform the magnetic

†Kongsberg Defence \& Aerospace. 


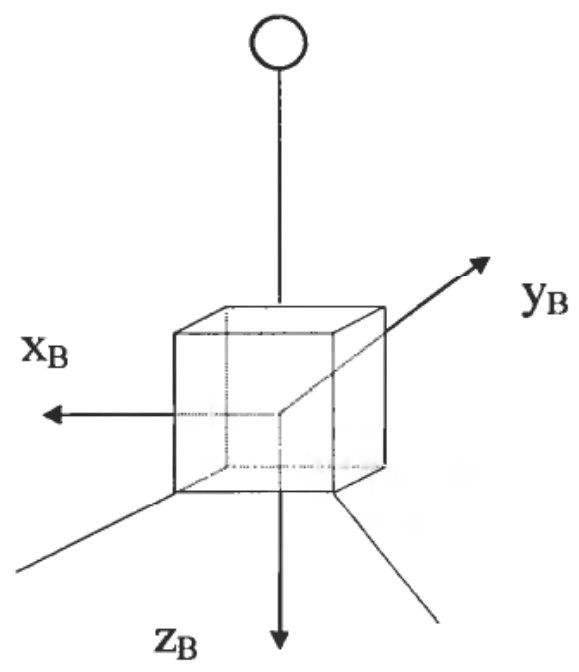

Figure 1. Proposed gravity gradient stabilised satellite.

torquing. The coils interact with the magnetic field of the earth and produce a satellite torque, which is used to control the rotation of the satellite. A problem is that both the direction and the strength of the geomagnetic field change and magnetic control become non-linear and time-invariant. Another problem is that the torque becomes zero when the coil's and earth's magnetic field align, which mean that azimuth become non-controllable over Equator.

Magnetic torquing may achieve attitude angles down to a few degrees. More accurate attitude control requires wheels, i.e., momentum wheels, reaction wheels or control torque gyros. A momentum wheel is a flywheel rotating at nominal speed, called a biased flywheel. The wheel speed changes according to the required control torque. A reaction wheel is a nonbiased flywheel, i.e., the wheel is nominally at rest. A control torque gyro is a gimballed biased flywheel. This paper deals with magnetic coils and reaction wheels only.

Hodgart et al. (1994) combined gravity gradient stabilisation and two magnetic coils to achieve relatively accurate pitch and roll control. Musser et al. (1989) combined gravity gradient stabilisation and magnetic coils and also showed that linear quadratic (LQ) control may be used to obtain three-axis stabilisation of a satellite. Bak et al. (1996), Suglo (1994) and Wisniewski (1996) all describe attitude control combining gravity gradient stabilisation, magnetic torquing and LQ control. This idea is also treated in Narheim et al. (1994). Cavallo et al. (1993) apply two magnetic coils and one reaction wheel controlled from a sliding mode strategy.

Wertz (1978) describes three satellites combining different actuators. SEASAT combine wheel control and gravity gradient stabilisation to achieve an accuracy of $0.5^{\circ}$ in all three axes. HCMM uses pitch wheel to achieve an accuracy of $1^{\circ}$ and magnetic torquing in roll also to an accuracy of $1^{\circ}$. A quarter-orbit coupling gives an accuracy of $2^{\circ}$ in azimuth. GEOS- 3 uses an extendable $6.5 \mathrm{~m}$ boom with a $45 \mathrm{~kg}$ end mass to achieve a large gravity gradient restoring torque which combined with a damper, provides a $1^{\circ}$ roll and pitch control and a quarter-orbit coupling that provide an azimuth control accuracy of $1.5^{\circ}$.

Section 2 discusses sensors and actuators that can be used to stabilise a low orbit 
satellite. Section 3 presents a mathematical model of the satellite and the actuators to be used of the satellite. Section 4 shows how the present low orbit satellite can be stabilised using gravity gradient stabilisation combined with coil and/or wheel control. Conclusions are found in section 5 .

\section{Sensors and Actuators}

\subsection{Sensors}

Horizon Sensors detect the horizon of the earth and thereby determine the local vertical direction. There are three types of practical horizon sensors: based on scanning, balanced radiation or edge tracking (Wertz 1990). Horizon sensors achieve accuracy down to $0.5^{\circ}$. Satellites that require better pointing accuracy, such as communication and weather satellites, apply star sensors and/or inertial navigation.

Star Sensors measure star co-ordinates in the spacecraft frame and provide attitude information when these observed co-ordinates are compared with known star directions obtained from a star catalogue. Star sensors may achieve accuracy down to a few arc-sec $\left(\right.$ arc-sec $\left.=1 / 3600^{\circ}\right)$.

Inertial Navigation makes use of gyros and accelerometers. For space flight, small gyro and accelerometer errors accumulate and periodic updates from an external reference source are required. Often a combination of gyros and star sensors are used.

Magnetometers detect both the direction and magnitude of the magnetic field. A magnetometer, combined with a global magnetic field model, will provide the satellite attitude. The magnetic field, however, which is highly non-linear, is not completely known and the accuracy of the magnetometers is limited to a few degrees (Wertz 1990). It is also a problem that the earth's magnetic field strength decreases with distance from the earth, so the use of magnetometers is generally limited to altitudes below $1000 \mathrm{~km}$.

Global Positioning System (GPS). Time, local position and velocity estimates are obtained from a GPS receiver.

\subsection{Actuators}

Magnetic Coil generates a torque that can be used to control the satellite attitude. The electromagnetic coils are normally mounted on the $x, y$ and $z$ facets of the satellite. Magnetic torquing does not require moving parts and may provide an attitude control down to a few degrees.

Reaction Wheel is a nonbiased flywheel, i.e., the wheel is nominally at rest. A wheel speed is generated depending on the required control torque. A reaction wheel will sooner or later saturate and a gas jet, magnetic coil or gravity gradient torque must be used to restore the momentum wheel speed to its nominal operating value, called momentum dumping. Since reaction wheels go through the zero-speed region, models of the torque and the friction characteristics may be needed to model accurate reaction wheel control.

\section{Mathematical Model of the Satellite}

The satellite consists of a gravity boom connected to a satellite body. The gravity boom has a tip mass of $2 \mathrm{~kg}$ to improve the gravity gradient stabilisation. The gravity boom is flexible, but is in this paper, as a first-order approximation, considered to be rigid. External actuators like electromagnetic coils and reaction wheels are added to 


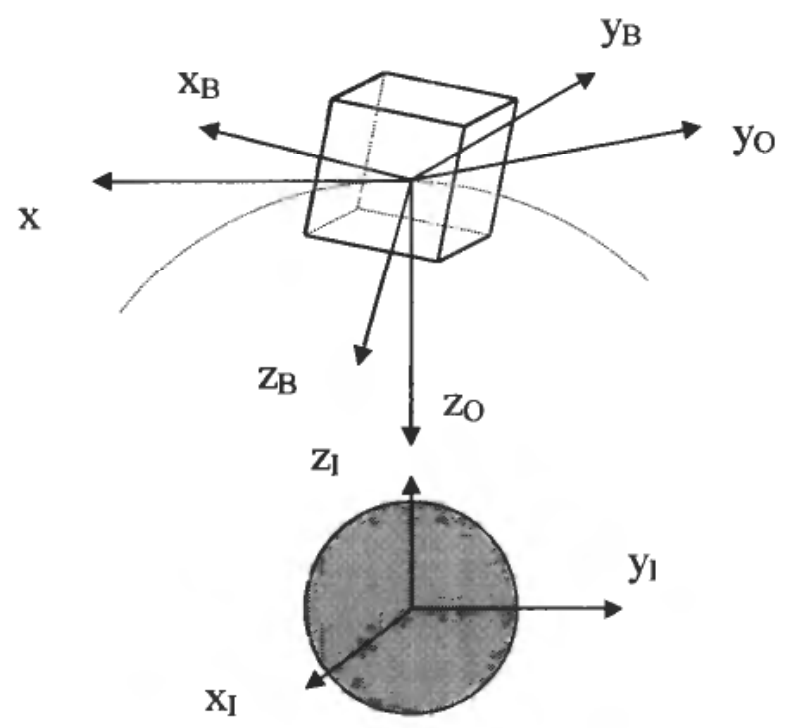

Figure 2. Co-ordinate system definition.

improve both the three-axis stabilisation and the pointing properties. Suitable mathematical models for these actuators are presented. The latter applied control methods require linear plants, and the non-linear mathematical satellite model is therefore linearised. The linearised model is modelled using MATLAB and several simulations are performed. The torque equation, assuming the satellite to be a stiff body, may be written (Skullestad 1995),

$$
J^{B} \dot{\omega}_{B / I}+{ }^{B} \omega_{B / I} \times\left(J^{B} \omega_{B / I}\right)={ }^{B} T
$$

where:

$J-$ moment of inertia matrix referred to frame B, i.e., $J=\operatorname{diag}\left(J_{x}, J_{y}, J_{z}\right)$

${ }^{B} \mathrm{~T}$-total torque acting on the satellite expressed in frame $\mathrm{B}$ components

${ }^{B} \omega_{B I I}$-angular velocity of frame B referred to frame I expressed in frame B components

The co-ordinate systems are defined in Fig. 2. The inertia reference frame (I-frame) is located at the centre of the earth and defined by the co-ordinates $x_{1}, y_{1}, z_{1}$, the $z_{1}$-axis is parallel to the rotation axis of the earth, the $\mathrm{x}_{\mathrm{I}}$-axis is pointing towards a fixstar (Soglo 1994). The body frame (B-frame) is located with its origo in the mass centre of the satellite and defined by the co-ordinates $x_{B}, y_{B}, z_{B}$. The $x_{B}$-axis is called the roll-axis, $y_{B}$ is called the pitch-axis, and $z_{B}$ is called the azimuth-axis (or yaw-axis). The body frame axis aligns the principal-axis of the rigid body, hence, the moment of inertia matrix is simplified to a diagonal inertia matrix (1). The orbit frame is located with origo in the mass centre of the satellite and defined by the co-ordinates $x_{0}, y_{0}, z_{0}$. The $x_{0}$-axis is parallel to the velocity vector of the satellite, $y_{0}$ is perpendicular to the orbit plane and $\mathrm{z}_{0}$ points toward the middle of the earth.

The angular velocity expressed in body co-ordinates referred to frame $\mathrm{I},{ }^{B} \omega_{B I I}$, may be written

$$
{ }^{B} \omega_{B I I}={ }^{B} \omega_{B / O}+{ }^{B} \omega_{O I I}={ }^{B} \omega_{B / O}+R_{B}^{O O}{ }^{O} \omega_{O I I}
$$


where

${ }^{B} \omega_{B I}$-defined in (1).

${ }^{B} \omega_{O I I}$-angular velocity of frame $\mathrm{O}$ referred frame $\mathrm{I}$, expressed in frame $\mathrm{B}$ components

${ }^{B} \omega_{B / O}$-angular velocity of frame $\mathrm{B}$ referred frame $\mathrm{O}$, expressed in frame $\mathrm{B}$ components.

${ }^{o} \omega_{B I}$-angular velocity of frame $\mathrm{B}$ referred frame $\mathrm{O}$, expressed in frame $\mathrm{O}$ components.

$R_{B}^{O}$-transforms the components of a vector expressed in frame $\mathrm{O}$ to components expressed in frame B. $R_{B}^{O}$ is defined in chapter 3.2.

The angular velocity of frame $\mathrm{O}$ referred frame I simply consists of the angular velocity of the satellite referred earth

$$
{ }^{o} \omega_{O / l}=\left[\begin{array}{lll}
0 & -\omega_{O} & 0
\end{array}\right]^{T}
$$

The angular velocity, $\omega_{o}$, can be expressed as (Wiesel 1989)

$$
\omega_{o}=\sqrt{\frac{\mu}{R_{c}^{3}}}
$$

Assuming further that the external torque consits of the gravity gradient torque, actuator torque, and the disturbance torque, then the torque may be expressed as

$$
{ }^{B} T={ }^{B} T_{G}+{ }^{B} T_{A}+{ }^{B} T_{D}
$$

${ }^{B} T_{G}$ - gravity gradient torque expressed in frame B components and defined in chapter 3.5 .

${ }^{B} T_{A}-$ actuator torque expressed in frame B components and defined in chapter 3.6 and 3.7.

${ }^{B} T_{D}$ - disturbance torque expressed in frame B components and defined in chapter 4 .

\subsection{Rotation matrix}

The transformation matrix or rotation matrix, $R_{O}^{B}$, transforms components expressed in frame $\mathrm{B}$ to components expressed in frame $\mathrm{O}$.

A vector ${ }^{B} \omega$ expressed in frame $\mathrm{B}$ components may be transformed to frame $\mathrm{O}$ components using (6)

$$
{ }^{o} \omega=R_{O}^{B B} \omega
$$

The rotation matrix, $R_{O}^{B}$, may be calculated using Euler angles, Euler parameters (Quarternions) or Euler-Rodrigues parameters. Euler-Rodrigues parameters will not be described in this paper.

\subsection{Euler angles}

The Euler angles may be expressed in 12 different ways, each of them giving a different rotation matrix, $R_{O}^{B}$. Both in missiles and in satellites the rotations defined in (7) are often used (Skullestad 1993),

$$
R_{O}^{B}=R_{z, \varphi} R_{y, \theta} R_{x, \phi}
$$

where

$$
\boldsymbol{R}_{z, \varphi} \text {-angular rotation } \psi \text { degrees about } \mathrm{z}_{\mathrm{O}} . \psi \text { is called the azimuth angle. }
$$


$R_{y, 0}$-angular rotation $\theta$ degrees about the new y-axis. $\theta$ is called the pitch angle.

$R_{x, \phi}$-angular rotation $\phi$ degrees about the new $\mathrm{z}$-axis. $\phi$ is called the roll angle.

The rotation matrix is defined as

$$
R_{z, \psi}=\left[\begin{array}{ccc}
c \psi & -s \psi & 0 \\
s \psi & c \psi & 0 \\
0 & 0 & 1
\end{array}\right], R_{y, \theta}=\left[\begin{array}{ccc}
c \theta & 0 & s \theta \\
0 & 1 & 0 \\
-s \theta & 0 & c \theta
\end{array}\right], R_{x, \phi}=\left[\begin{array}{ccc}
1 & 0 & 0 \\
0 & c \phi & -s \phi \\
0 & s \phi & c \phi
\end{array}\right]
$$

The notation $\mathrm{s}=\sin$ and $\mathrm{c}=\cos$ is used.

Multiplying the above matrices in the order given in (7) gives the rotation matrix

$$
R_{O}^{B}=\left[\begin{array}{ccc}
c \psi c 0 & -s \psi c \phi+c \psi s 0 s \phi & s \psi s \phi+c \psi c \phi s \theta \\
s \psi c \theta & c \psi c \phi+s \phi s \theta s \psi & -c \psi s \phi+s \theta s \psi c \phi \\
-s \theta & c \theta s \phi & c \theta c \phi
\end{array}\right]
$$

(9) becomes singular for $\mathrm{c} \theta=0$, i.e., $\theta= \pm 90^{\circ}$. If the pitch angle becomes $\geq 90^{\circ}$, additional logic should be included. Euler parameters may be applied without special attention to angles $\geq 90^{\circ} . R_{B}^{O}=\left(R_{O}^{B}\right)^{-1}=\left(R_{O}^{R}\right)^{T}$, due to symmetry properties of the rotation matrix.

\subsection{Euler parameter}

The Euler parameters may be defined as Hughes (1986)

$$
\left(\varepsilon_{1}, \varepsilon_{2}, \varepsilon_{3}\right)^{\mathrm{T}}=\operatorname{asin} \frac{\theta}{2} \text { and } \eta=\cos \frac{\theta}{2}
$$

where $\theta$ is an angular rotation around a unit vector a. The parameters $\varepsilon_{1}, \varepsilon_{2}, \varepsilon_{3}$ and $\eta$ are called Euler parameters. An angular displacement is then specified by $(\varepsilon, \eta)$. Note that $\varepsilon_{1}+\varepsilon_{2}+\varepsilon_{3}+\eta=1$. $(\varepsilon, \eta)$ are also called quaternions.

The rotation matrix based on Euler parameters may be written (Soglo 1994),

$$
R_{O}^{R}=I+2 S^{2}(\varepsilon)+2 \eta S(\varepsilon)
$$

where $S(\varepsilon)=S^{T}(\varepsilon)$ is a skew-symmetric matrix. Assuming $\varepsilon$ to be a column vector, defined as $\varepsilon=\left[\begin{array}{lll}\varepsilon_{1} & \varepsilon_{2} & \varepsilon_{3}\end{array}\right]^{T}$, then $\mathrm{S}(\varepsilon)$ may be expressed as

$$
S(\varepsilon)=\left[\begin{array}{ccc}
0 & -\varepsilon_{3} & \varepsilon_{2} \\
\varepsilon_{3} & 0 & -\varepsilon_{1} \\
-\varepsilon_{2} & \varepsilon_{1} & 0
\end{array}\right]
$$

(12) is simply the matrix representation of $\varepsilon \times$.

$R_{O}^{B}$ may be expressed in matrix form as Hughes (1986)

$$
R_{O}^{B}=\left[\begin{array}{ccc}
\eta^{2}+\varepsilon_{1}^{2}-\varepsilon_{2}^{2}-\varepsilon_{3}^{2} & 2\left(\varepsilon_{1} \varepsilon_{2}-\eta \varepsilon_{3}\right) & 2\left(\varepsilon_{1} \varepsilon_{3}-\eta \varepsilon_{2}\right) \\
2\left(\varepsilon_{1} \varepsilon_{2}+\eta \varepsilon_{3}\right) & \eta^{2}-\varepsilon_{11}^{2}-\varepsilon_{2}^{2}-\varepsilon_{3}^{2} & 2\left(\varepsilon_{2} \varepsilon_{3}-\eta \varepsilon_{1}\right) \\
2\left(\varepsilon_{1} \varepsilon_{3}-\eta \varepsilon_{2}\right) & 2\left(\varepsilon_{2} \varepsilon_{3}+\eta \varepsilon_{1}\right) & \eta^{2}-\varepsilon_{1}^{2}-\varepsilon_{2}^{2}-\varepsilon_{3}^{2}
\end{array}\right]
$$

The Euler parameters may be determined from Egeland (1993).

$$
\dot{\eta}=-\frac{1}{2} \varepsilon^{T B} \omega_{B / O}
$$




$$
\dot{\varepsilon}=\frac{1}{2}(\eta I+S(\varepsilon))^{B} \omega_{B / O}
$$

\subsection{Transformation between Euler parameters and Euler angles}

Given the rotation matrix, $R_{O}^{B}$, expressed in Euler parameters. The Euler angles may be calculated from (15) (Fossen 1994),

$$
\begin{aligned}
& \theta=-a \sin \left(r_{31}\right) \\
& \phi=a \tan \left(r_{32}, r_{33}\right) \quad \theta \neq \pm 90^{\circ} \\
& \psi=a \tan \left(\frac{r_{21}}{r_{11}}\right)
\end{aligned}
$$

Given the rotation matrix, $R_{O}^{B}$, expressed in Euler angles. The Euler parameters may now be calculated from (16)

$$
\begin{aligned}
\eta^{2}+\varepsilon_{1}^{2}+\varepsilon_{2}^{2}+\varepsilon_{3}^{2} & =1 \\
2\left(\varepsilon_{1} \varepsilon_{3}-\eta \varepsilon_{2}\right) & =r_{13} \\
2\left(\varepsilon_{2} \varepsilon_{3}-\eta \varepsilon_{12}\right) & =r_{23} \\
\eta^{2}-\varepsilon_{1}^{2}-\varepsilon_{2}^{2}+\varepsilon_{3}^{2} & =r_{33}
\end{aligned}
$$

\subsection{Gravity Gradient Torque}

A body with non-uniform mass-distribution will, when exposed to a quadratic decaying gravitational field, be influenced by torque. For most spacecraft situations, the following simplifications can be made (Hughes 1986).

- Only the gravitational field from the earth is considered.

- The satellite is small compared to its distance from the mass centre of the earth.

- The satellite consists of a single body.

A satellite in the gravitational field of the earth is influenced by a gravitational force, given by Newton's second law (Hughes 1986)

$$
\vec{f}=-\mu \int_{B} \frac{\vec{R}}{R^{3}} d m
$$

where $\mu=\gamma \mathrm{m}_{\text {earth }}, \gamma$ is the universal gravitational constant, mearth is the mass of the earth, $B$ denotes the body of the satellite, $\mathrm{R}$ is the location of the mass element $\mathrm{dm}$ relative to the centre of the earth and

$$
\mathrm{R}=|\vec{R}| .
$$

Inserting values gives $\mu=3.986 \times 10^{14} \mathrm{Nm}^{2} / \mathrm{kg}$. The gravitational torque around the centre of the mass is

$$
T_{G}=-\mu \int_{B} \frac{\vec{r} \times \vec{R}}{R^{3}} d m
$$

$\vec{R}=\vec{R}_{c}+\vec{r}$, where $\vec{R}_{c}$ is the location of the mass centre of the satellite and $\vec{r}$ is the distance from the mass centre of the body to the actual mass element. The satellite is small compared to its distance from the mass centre of the earth. To simplify the above expression a binomical series expansion of $\mathrm{R}^{-3}$ is used (Narheim et al. 1994), 


$$
R^{-3}=R_{c}^{-3}\left(1-3 \frac{\vec{r} \times \vec{R}_{c}}{R_{c}^{2}}+\ldots\right)
$$

Finally the gravitational field in frame B coordinates can be expressed as (Hughes 1986),

$$
{ }^{B} T_{G}=3\left(\frac{\mu}{R_{c}^{3}}\right) r_{3} \times J r_{3}
$$

where $\mathrm{J}$ is the inertia matrix and $\mathrm{r}_{3}$ the third column in $R_{B}^{O}$.

The gravitational torque, in frame B components, can be written (Hughes 1986)

$$
{ }^{B} T_{G}=3\left(\frac{\mu}{R_{c}^{3}}\right)\left(\begin{array}{l}
\left(\left(J_{33}-J_{22}\right) r_{23} r_{33}+J_{23}\left(r_{23}^{2}-r_{33}^{2}\right)+J_{31} r_{13} r_{23}-J_{12} r_{33} r_{13}\right. \\
\left(\left(J_{11}-J_{33}\right) r_{33} r_{13}+J_{31}\left(r_{33}^{2}-r_{13}^{2}\right)+J_{12} r_{23} r_{33}-J_{23} r_{13} r_{23}\right. \\
\left(\left(J_{22}-J_{11}\right) r_{13} r_{23}+J_{12}\left(r_{13}^{2}-r_{23}^{2}\right)+J_{23} r_{33} r_{13}-J_{31} r_{23} r_{33}\right.
\end{array}\right)
$$

where $\mathbf{J}=\mathrm{J}_{\mathrm{ij}}$ and $R_{B}^{O}=\mathrm{r}_{\mathrm{ij}}$. (22)

If the principal axes are chosen as the reference axis in frame B, (21) simplifies to

$$
{ }^{B} T_{G}=3 \omega_{O}^{2}\left[\begin{array}{l}
\left(J_{z}-J_{y}\right) r_{23} r_{33} \\
\left(J_{x}-J_{z}\right) r_{33} r_{13} \\
\left(J_{y}-J_{x}\right) r_{13} r_{23}
\end{array}\right]
$$

The gravitation torque may be expressed, using Euler angles, i.e., $r_{13}=-\sin 0$, $r_{23}=\cos \theta \sin \phi$ and $r_{33}=\cos \theta \cos \phi$ (Soglo 1994),

$$
\left[\begin{array}{c}
{ }^{B} T_{G x} \\
{ }^{B} T_{G y} \\
{ }^{B} T_{G z}
\end{array}\right]=3 \omega_{O}^{2}\left[\begin{array}{c}
\left(J_{z}-J_{y}\right) \sin \phi \cos \phi(\cos \theta)^{2} \\
\left(J_{z}-J_{x}\right) \cos \phi \sin \theta \cos \theta \\
\left(J_{x}-J_{y}\right) \sin \phi \sin \theta \cos \theta
\end{array}\right]
$$

\subsection{Magnetic Control Torque}

A spacecraft exposed to a magnetic field will, supposing that the spacecraft has a magnetic coil, be influenced by a magnetic torque. Denoting the external magnetic flux density by $\mathrm{B}$ and the magnetic moment by $\mathrm{m}$, the torque acting on the spacecraft can be written

$$
{ }^{B} T={ }^{B} m \times{ }^{B} B
$$

Controlling the magnetic moment ${ }^{B} \mathrm{~m}$, e.g., using magnetic coils, provide a mean of performing attitude control. The magnetic torque will according to (24) act perpendicular to the magnetic moment vector ${ }^{\mathrm{B}} \mathrm{m}$ and the magnetic field vector ${ }^{\mathrm{B}} \mathrm{B}$. Three perpendicular electromagnetic air cored coils are normally applied to fully control the satellite.

\subsubsection{Magnetic Field}

A model of the magnetic field of the earth should be established, before the magnetic moment acting on the satellite can be determined. The magnetic field varies strongly at large altitudes. Lower altitudes, approximately $1200 \mathrm{~km}$ or less, have a relatively constant magnetic field. Hughes (1986) suggested using a dipole moment as the earth's magnetic field at low altitudes. The magnetic field points towards the Southern Hemisphere, i.e., $\mathbf{z}_{\mathrm{m}}$ points towards the magnetic South Pole. The end of the earth's dipole in the Northern Hemisphere is at $78.6^{\circ} \mathrm{N}, 289.3^{\circ} \mathrm{E}$. Fig. 3 shows the geomagnetic 


\section{Satellite}

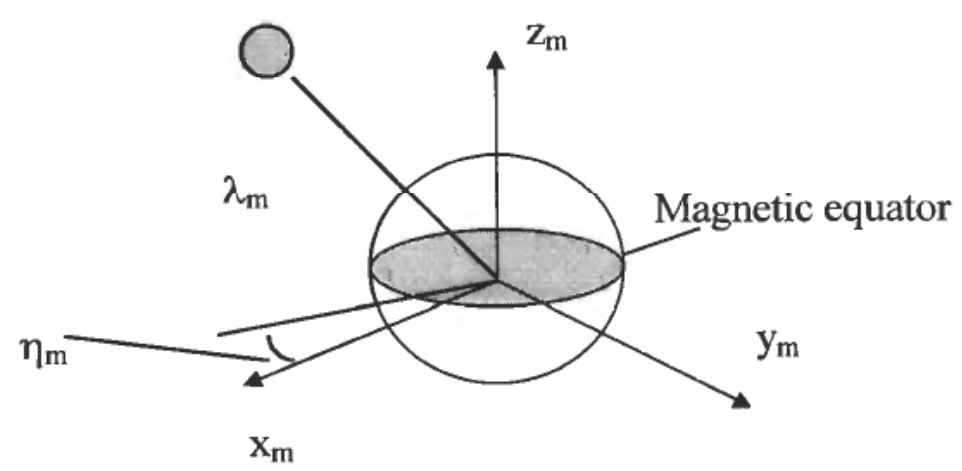

Figure 3. Magnetic field co-ordinate definition.

reference frame $\mathrm{m}$ (Soglo 1994). The dipole moment, describing the magnetic field of the earth, can be expressed using the magnetic potential (Hughes 1986)

$$
\phi_{m}=-\frac{\mu_{m}}{R_{c}^{2}} \sin \lambda_{m}
$$

where $R_{c}$ is the distance from the centre of the earth, $\lambda_{M}$ is the latitude with respect to the geomagnetic equatorial plane and

$$
\mu_{m}=\frac{1}{4 \pi} \times 10^{17}=8.10^{15} \mathrm{Wbm}
$$

is the earth's dipole strength. The magnetic flux density is then calculated from

$$
B=-\nabla \phi_{m}
$$

where $\nabla$ is the vector-gradient operator.

The magnetic flux density expressed in the geomagnetic reference frame (Hughes 1986),

$$
{ }^{m} B=-B_{O}\left[\begin{array}{c}
3 \sin \lambda_{m} \cos \lambda_{m} \cos \eta_{\mathrm{m}} \\
3 \sin \lambda_{m} \cos \lambda_{m} \sin \eta_{\mathrm{m}} \\
3\left(\sin \lambda_{m}\right)^{2}-1
\end{array}\right]
$$

where $R_{c}=7571 \times 10^{3} \mathrm{~m}$ at an altitude of $1200 \mathrm{~km}$ and

$$
B_{O}=\frac{\mu_{m}}{R_{c}^{3}}=1.843 \times 10^{-5} \mathrm{~Wb} / \mathrm{m}^{2}
$$

The magnetic fiux density may be expressed in frame B components, using the rotation matrices

$$
{ }^{B} \mathrm{~B}=\boldsymbol{R}_{B}^{m m} \mathrm{~B}=\boldsymbol{R}_{B}^{O} \boldsymbol{R}_{O}^{m m} \mathrm{~B}=\boldsymbol{R}_{\mathrm{B}}^{O}{ }^{o} \mathrm{~B}
$$

$R_{m}^{O}$ can be defined by first rotating $\lambda_{\mathrm{m}}{ }^{\circ}$ around the $\mathrm{z}_{\mathrm{m}}$-axis, then a rotation $\eta_{\mathrm{m}}{ }^{\circ}$ around the new $y$-axis, then a rotation $-90^{\circ}$ around the same $y$-axis and finally a rotation $\delta_{\mathrm{m}}{ }^{\circ}$ around the new z-axis (Soglo 1994). 


$$
\begin{aligned}
& R_{m}^{O} \\
& =\left[\begin{array}{ccc}
-\cos \eta_{m} \sin \lambda_{m} \cos \delta_{m}-\sin \eta_{m} \sin \delta_{m} & \cos \eta_{m} \sin \lambda_{m} \sin \delta_{m}-\sin \eta_{m} \cos \delta_{m} & -\cos \eta_{m} \cos \lambda_{m} \\
-\cos \eta_{m} \sin \delta_{m}-\sin \eta_{m} \sin \lambda_{m} \cos \delta_{m} & \cos \eta_{m} \cos \delta_{m}+\sin \eta_{m} \sin \lambda_{m} \sin \delta_{m} & -\sin \eta_{m} \cos \lambda_{m} \\
\cos \lambda_{m} \cos \delta_{m} & -\cos \lambda_{m} \sin \delta_{m} & -\sin \lambda_{m}
\end{array}\right]
\end{aligned}
$$

$\delta_{\mathrm{m}}$ is the angle between the satcllite's velocity vector and the plane formed by $\mathrm{z}_{\mathrm{m}}$ and $\mathrm{z}_{\mathrm{o}}$. The magnetic field is symmetric around $\mathrm{z}_{\mathrm{m}}$ and hence $\eta_{\mathrm{m}}$ can be removed from $R_{m}^{O}$.

The magnetic and geographic poles of the earth do not align; i.e., $\mathrm{z}_{\mathrm{m}}$ is not parallel to the rotation axis, $\mathrm{z}_{\mathrm{l}}$, of the earth. Both the angle $\lambda_{\mathrm{m}}$ and $\delta_{\mathrm{m}}$ will, seen from frame $\mathrm{I}$, vary due to earth rotation. The rotation of the earth, however, is much less than the angular velocity of the satellite, and as a first approximation the magnetic north pole is assumed to coincide with geographic North Pole. The magnetic flux density expressed in frame $\mathbf{O}$ can be written

$$
{ }^{o} B=R_{O}^{m m} B=\left(R_{m}^{O}\right)^{T m} B=B_{0}\left[\begin{array}{c}
\cos \lambda_{m} \cos \delta_{m} \\
-\cos \lambda_{m} \sin \delta_{m} \\
2 \sin \lambda_{m}
\end{array}\right]
$$

Assuming further that the satellite moves over the poles, i.e., $\delta_{\mathrm{m}}=0(30)$, simplifies to

$$
{ }^{o} B=R_{O}^{m m} B=B_{0}\left[\begin{array}{c}
\cos \lambda_{m} \\
0 \\
2 \sin \lambda_{m}
\end{array}\right]
$$

\subsubsection{Magnetic Control Torque}

The coils are mounted on the $\mathrm{x}, \mathrm{y}$ and $\mathrm{z}$ facets of the satellite body. The magnetic moment resulting from a $\mathrm{N}$ turns air cored coil with current $\mathrm{I}$ and coil area A may be written (Wertz 1990)

$$
{ }^{\mathrm{B}} \boldsymbol{m}=I A N
$$

Adding (32) to (24) and expressing the magnetic control torque, acting on the satellite, in frame $\mathrm{B}$ components gives

$$
{ }^{B} T_{m}=\left[\begin{array}{l}
N A i_{x} \\
N A i_{y} \\
N_{z} A i_{z}
\end{array}\right] \times{ }^{B} B
$$

where $i_{x}, i_{y}$ and $i_{z}$ are the coil currents.

The electrical model of a coil can be written (Hayt et al. 1963),

$$
L \frac{d i}{d t}+R i-e=U
$$

where $L$ is the coil inductance, $R$ is the coil resistance, $i$ is the current, e is the back EMF and $U$ is the applied voltage. In frame B components (34) may be written

$$
\begin{aligned}
& i_{x}=-\frac{R_{x}}{L} i_{x}+\frac{1}{L} e_{x}+\frac{1}{L} U_{x} \\
& i_{y}=-\frac{R_{y}}{L} i_{y}+\frac{1}{L} e_{y}+\frac{1}{L} U_{y}
\end{aligned}
$$




$$
\dot{i}_{z}=-\frac{R_{z}}{L} i_{z}+\frac{1}{L} e_{z}+\frac{1}{L} U_{z}
$$

The back EMF in the x-coil may be expressed as (Soglo 1994),

$$
\begin{aligned}
e_{x} & =-\frac{d}{d t}\left(N_{x} A_{x}\left({ }^{B} B^{T} x_{B}\right)\right) \\
& =-N_{x} A_{x}\left(\frac{d}{d t}\left({ }^{o} B^{T} R_{O}^{B}\right) x_{B}\right) \\
& =-N_{x} A_{x}\left(\left({ }^{O} B^{T} \dot{R}_{O}^{B}+{ }^{o} \dot{B}^{T} R_{O}^{B}\right) x_{B}\right)
\end{aligned}
$$

where $\mathrm{x}_{\mathrm{B}}=\left[\begin{array}{lll}1 & 0 & 0\end{array}\right]^{\mathrm{T}}$. Note that $\dot{R}_{O}^{B}$ can be expressed as

$$
\dot{R}_{O}^{B}=R_{O}^{B} S\left({ }^{B} \omega_{B / O}\right)
$$

Based on the above expressions the back EMF of the coils can be expressed in frame B components as

$$
\begin{aligned}
& e_{x}=-N_{x} A_{x}\left(\left({ }^{O} \dot{B}^{T} R_{O}^{B}+{ }^{B} B^{T} S\left({ }^{B} \omega_{B / O}\right)\right) x_{B}\right) \\
& e_{y}=-N_{y} A_{y}\left(\left({ }^{O} \dot{B}^{T} R_{O}^{B}+{ }^{B} B^{T} S\left({ }^{B} \omega_{B / O}\right)\right) y_{B}\right) \\
& e_{z}=-N_{z} A_{z}\left(\left({ }^{O} \dot{B}^{T} R_{O}^{B}+{ }^{B} B^{T} S\left({ }^{B} \omega_{B / O}\right)\right) z_{B}\right)
\end{aligned}
$$

where $\mathrm{x}_{\mathrm{B}}=\left[\begin{array}{lll}1 & 0 & 0\end{array}\right]^{\mathrm{T}}, \mathrm{y}_{\mathrm{B}}=\left[\begin{array}{lll}0 & 1 & 0\end{array}\right]^{\mathrm{T}}$ and $\mathrm{z}_{\mathrm{B}}=\left[\begin{array}{lll}0 & 0 & 1\end{array}\right]^{\mathrm{T}}$.

Differentiating (31) with respect to time yields ${ }^{\circ} \dot{B}$

$$
{ }^{o} \dot{B}=B_{0}\left[\begin{array}{c}
-\dot{\lambda}_{m} \sin \lambda_{m} \\
0 \\
2 \dot{\lambda}_{m} \cos \lambda_{m}
\end{array}\right]
$$

$\dot{\lambda}_{m}$ is the angular velocity of the satellite seen from frame $\mathrm{I}$, also assuming that frame $\mathrm{m}$ and I coincide, i.e., $\dot{\lambda}_{m}=\omega_{0}$, hence (39) may be written

$$
{ }^{o} \dot{B}=\omega_{o} B_{0}\left[\begin{array}{c}
-\sin \lambda_{m} \\
0 \\
2 \cos \lambda_{m}
\end{array}\right]
$$

\subsection{Reaction Wheel}

A reaction wheel consists of a flywheel rigidly mounted in the satellite and is designed to operate from a nonbiased or zero torque. An electric motor forces the flywheel to rotate at an angular rate of $\omega_{\mathrm{w}}$ relative to the satellite. The torque of the reaction wheel will cause the satellite to rotate at a rate $\omega_{\mathrm{s}}$ with respect to inertial space. The dynamic of a reaction wheel can be included as an additional term in Euler's equation and is given by Kapland (1976)

$$
{ }^{B}\left(\frac{d H}{d t}\right)+\omega \times H={ }^{B} T
$$

where ${ }^{\mathrm{B}} \mathrm{T}$ is the total torque acting on the satellite expressed in frame B components, $\mathrm{H}$ is the total angular momentum including the wheel, i.e., $\mathrm{H}=\mathrm{H}_{\mathrm{S}}+\mathrm{H}_{\mathrm{w}} . \mathrm{H}_{\mathrm{S}}$ is the angular momentum of the satellite and $\mathrm{H}_{W}$ is the angular momentum of the wheel.

Since the flywheel will be rotating at an angular rate of $\omega_{\mathrm{W}}-\omega_{\mathrm{S}}$ in inertial space, conservation of angular momentum requires 


$$
\boldsymbol{H}=\mathbf{0}=\boldsymbol{J}_{\mathrm{W}}\left(\omega_{\mathrm{w}}-\omega_{\mathrm{S}}\right)-J_{S} \omega_{S}
$$

The satellite rate, given the wheel rate, can be found from (42)

$$
\omega_{S}=-\frac{J_{W} \omega_{W}}{J_{S}+J_{W}}
$$

where $\mathbf{J}_{\mathrm{W}}$ is the moment of inertia of the wheel, $\mathbf{J}_{\mathrm{S}}$ is the moment of inertia of the satellite, $\omega_{\mathrm{W}}$ is the angular rate of the wheel, and $\omega_{\mathrm{S}}$ is the angular rate of the satellite. The same momentum can be achieved with a small high-speed flywheel as with a large low-speed one. The high-speed wheel has the disadvantages of greater wear on the bearings, which may shorten its lifetime, but at the benefit of a lower weight. Since the reaction wheel operates around zero angular velocity, a model of the reaction wheel torque and the friction characteristics may be needed to model an accurate control system. Such a detailed study is not treated here.

Reaction wheels may be saturated from undesirable momentum terms, and momentum dumping (desaturation) using an auxiliary control system are required. Thrusters are often used to desaturate wheels, but motor fuels are required. Gravity gradient torque and/or magnetic coils may be a better solution in low earth orbit. The torques both from the gravity gradient stabilisation and magnetic fields are small and the desaturation time-constant is of the order of an orbit period. Thrusters, however, allow a much faster desaturation.

\subsection{Complete Mathematical Model}

(1) describe the rotational motion of the satellite. (5) describe the additional torques, like gravitational torque, actuator torque and disturbance torque, acting on the satellite. Adding (5) to (1) gives the complete torque equation for the satellite

$$
{ }^{B} \dot{\omega}_{B / I}=J^{-1}\left(-{ }^{B} \omega_{B / I} \times\left(J^{B} \omega_{B / I}\right)+{ }^{B} T_{G}+{ }^{B} T_{A}+{ }^{B} T_{D}\right)
$$

${ }^{B} T_{G}$ is given in (23). ${ }^{B} T_{A}$ is the actuator torque, when using magnetic coils, given in (33). A model of the coil current is shown in (34). Soglo 1994 shows that the back EMF in the coils is small and can be ignored. (34) therefore simplifies to

$$
i=-\frac{R}{L} i+\frac{1}{L} U
$$

\subsection{Linearised Model}

(1) may be expressed in frame B components (along the principal body axes) as

$$
\begin{aligned}
& J_{x} \dot{\omega}_{x}=\left(J_{y}-J_{z}\right) \omega_{y} \omega_{z}+T_{x} \\
& J_{y} \dot{\omega}_{y}=\left(J_{z}-J_{x}\right) \omega_{x} \omega_{z}+T_{y} \\
& J_{z} \dot{\omega}_{z}=\left(J_{x}-J_{y}\right) \omega_{x} \omega_{y}+T_{z}
\end{aligned}
$$

where $\omega_{\mathrm{x}}, \omega_{\mathrm{y}}, \omega_{\mathrm{z}}$ are the angular velocities of frame B, expressed in frame B components. $J_{x}, J_{y}, J_{z}$ are the moment of inertias in frame $B$ and $T_{x}, T_{y}, T_{z}$ are the additional torques expressed in frame $\mathrm{B}$ components. Attitude control requires the angular position of frame B with respect to frame I, i.e., locally horizontal axes. These angles may be described by three Euler angles, as described in chapter 3.2. Euler angles should be used with care for angles $\geq 90^{\circ}$, but perform well for small angles and are chosen in the following simulations. 
Since $\omega_{\mathrm{x}}, \omega_{\mathrm{y}}, \omega_{\mathrm{z}}$ are the angular velocities with respect to frame I, expressed in frame B components and $0, \phi, \psi$ are the Euler angles that measure the angles of the body-axes with respect to locally horizontal axes, the relationship between body angular velocities and Euler angular velocities may be expressed as (Bryson 1994),

$$
\left[\begin{array}{c}
\omega_{x} \\
\omega_{y} \\
\omega_{z}
\end{array}\right]=\left[\begin{array}{c}
\dot{\phi} \\
0 \\
0
\end{array}\right]+R_{x, \phi}\left[\begin{array}{l}
0 \\
\dot{\theta} \\
0
\end{array}\right]+R_{x, \phi} R_{y, 0}\left[\begin{array}{l}
0 \\
0 \\
\dot{\psi}
\end{array}\right]+R_{x, \phi} R_{y, 0} R_{z, \psi}{ }^{o} \omega_{O I I}
$$

where $\mathbf{R}_{\mathrm{x}, \phi}, \mathbf{R}_{\mathrm{y}, 0}, \mathbf{R}_{\mathrm{z}, \psi}$ are defined in (8) and ${ }^{o} \omega_{\text {oI }}$ is defined in (3).

If $\phi, 0, \psi$ are small in magnitude and also the non-linear terms $\dot{\psi} 0, \dot{\psi} \phi, \dot{\theta} \phi$ are small, (47) may be approximated as

$$
\begin{aligned}
& \omega_{x} \cong \dot{\phi}-\psi \omega_{O} \\
& \omega_{y}=\dot{\theta}-\omega_{o} \\
& \omega_{z}=\dot{\psi}+\phi \omega_{O}
\end{aligned}
$$

\subsubsection{Gravity Gradient Torque}

The gravity gradient torque, using Euler angles, is given by (23). If $\phi$ and 0 are small (23) simplifies to

$$
\left[\begin{array}{c}
{ }^{B} T_{G x} \\
{ }^{B} T_{G y} T_{G z}
\end{array}\right]=-3 \omega_{O}^{2}\left[\begin{array}{c}
\left(J_{y}-J_{z}\right) \phi \\
\left(J_{x}-J_{z}\right) \theta \\
0
\end{array}\right]
$$

The torque equation including the gravity gradient torque, i.e., adding (49) to (46) become

$$
\begin{aligned}
& J_{x} \dot{\omega}_{x}=\left(J_{y}-J_{z}\right) \omega_{y} \omega_{z}-3 \omega_{O}^{2}\left(J_{y}-J_{z}\right) \phi+T_{x} \\
& J_{y} \dot{\omega}_{y}=\left(J_{z}-J_{x}\right) \omega_{x} \omega_{z}-3 \omega_{O}^{2}\left(J_{z}-J_{x}\right) 0+T_{y} \\
& J_{z} \dot{\omega}_{z}=\left(J_{x}-J_{y}\right) \omega_{x} \omega_{y}+T_{z}
\end{aligned}
$$

Inserting (48) into (50) gives

$$
\begin{aligned}
& J_{x} \ddot{\varphi}=\omega_{O}\left(J_{x}-J_{y}+J_{z}\right) \dot{\psi}-4 \omega_{O}^{2}\left(J_{y}-J_{z}\right) \varphi+T_{x} \\
& J_{y} \ddot{\theta}=-3 \omega_{O}^{2}\left(J_{x}-J_{z}\right) \theta+T_{y} \\
& J_{z} \ddot{\psi}=-\omega_{O}\left(J_{x}-J_{y}+J_{z}\right) \dot{\varphi}-\omega_{O}^{2}\left(J_{y}-J_{x}\right) \psi+T_{z}
\end{aligned}
$$

The terms $\omega_{O}\left(J_{x}-J_{y}+J_{z}\right) \dot{\psi}$ and $\omega_{O}\left(J_{x}-J_{y}+J_{x}\right) \dot{\varphi}$ are known as gyroscopic coupling terms and arise from the rotation of the locally horizontal axes at orbit rate $\omega_{0}$. (51, $52,53)$ represent the linearized small angle satellite model, including the gravity stabilisation torque.

\subsubsection{Stabilisation of the Satellite}

The gravitational torque is small, but will to some extent stabilise the satellite, providing that proper moment of inertia conditions are chosen. The stability properties of a gravitational stabilised satellite are outlined below. 


\section{Pitch}

The characteristic of equation pitch, (52), Laplace transformed becomes

$$
s^{2}+3 \omega_{O}^{2} \frac{J_{x}-J_{z}}{J_{y}}=0
$$

If $\mathbf{J}_{\mathbf{x}}>\mathbf{J}_{\mathbf{z}}$, the system becomes a simple harmonic oscillator. The system is stable, but has undamped oscillation at a frequency

$$
\omega_{p}=\omega_{o} \sqrt{\frac{3\left(J_{x}-J_{z}\right)}{J_{y}}}
$$

$\omega_{\mathrm{p}}$ is called the pitch libration frequency. $\mathrm{J}_{\mathrm{x}}<\mathrm{J}_{\mathrm{z}}$ gives an unstable system and the satellite will swing away from equilibrium when disturbed.

\section{Roll/Azimuth}

The roll and azimuth equations (51) and (53) are coupled. The Laplace transform of (51) and (53) may be written (Bryson 1994),

$$
\left[\begin{array}{cc}
s^{2}+4 \omega_{O}^{2} k_{x} & \left(k_{x}-1\right) \omega_{O} s \\
-\left(k_{z}-1\right) \omega_{O} s & s^{2}+k_{z} \omega_{O}^{2}
\end{array}\right]\left[\begin{array}{l}
\varphi(s) \\
\psi(s)
\end{array}\right]=\left[\begin{array}{l}
\frac{T_{x}}{J_{x}} \\
\frac{T_{z}}{J_{z}}
\end{array}\right]
$$

where

$$
k_{x}=\frac{J_{y}-J_{z}}{J_{x}}, k_{z}=\frac{J_{y}-J_{x}}{J_{z}}
$$

The characteristic equation of roll and azimuth becomes

$$
\left(\frac{s}{\omega_{o}}\right)^{4}+\left(3 k_{x}+k_{x} k_{z}+1\right)\left(\frac{s}{\omega_{o}}\right)^{2}+4 k_{x} k_{z}=0
$$

Roll and azimuth are oscillatory, but stable for $\mathrm{k}_{\mathrm{x}}>0$ and $\mathrm{k}_{\mathrm{z}}>0$, as well as over a small region when $\mathrm{k}_{\mathrm{x}}<0$ and $\mathrm{k}_{\mathrm{z}}<0$, elsewhere the motions are unstable.

These requirements combined with the earlier requirement that $J_{x}>J_{z}$ for pitch stability, give the complete stability of the gravity gradient satellite equilibria.

It is preferable that

$$
\mathrm{k}_{\mathrm{x}}>\mathrm{k}_{\mathrm{z}}>0 \Leftrightarrow \mathrm{J}_{\mathrm{y}}>\mathrm{J}_{\mathrm{x}}>\mathrm{J}_{\mathrm{z}}
$$

This orientation corresponds to the minimum total energy configuration for a gravity gradient satellite. In the presence of energy dissipation, this is the only stable region.

\subsubsection{Reaction Wheel Torque}

(41) describes the rotational motion of the satellite including reaction wheels. (41) may be expressed as

$$
{ }^{I}\left(\frac{d H}{d t}\right)={ }^{B}\left(\frac{d H_{s}}{d t}\right)+{ }^{B}\left(\frac{d H_{W}}{d t}\right)+\omega \times\left(H_{S}+H_{W}\right)+{ }^{B} T_{D}
$$

Writing (59) in component form, ignoring the disturbance torque, ${ }^{\mathrm{B}} \mathrm{T}_{\mathrm{D}}$, gives

$$
\begin{aligned}
& J_{x} \dot{\omega}=\left(J_{y}-J_{z}\right) \omega_{y} \omega_{z}+\dot{h}_{W_{x}}+\omega_{y} h_{W_{z}}-\omega_{z} h_{W y} \\
& J_{y} \dot{\omega}_{y}=\left(J_{z}-J_{x}\right) \omega_{x} \omega_{z}+\dot{h}_{W_{y}}+\omega_{x} h_{W_{z}}-\omega_{z} h_{W_{x}} \\
& J_{z} \dot{\omega}_{z}=\left(J_{y}-J_{x}\right) \omega_{x} \omega_{y}+\dot{h}_{W_{z}}+\omega_{x} h_{W_{y}}-\omega_{y} h_{W_{x}}
\end{aligned}
$$


Using the small angle approximation given in (48) and adding the gravity gradient torque (49) gives

$$
\begin{aligned}
& J_{x} \ddot{\varphi}=4 \omega_{O}^{2}\left(J_{y}-J_{z}\right) \varphi+\omega_{O}\left(J_{x}-J_{y}+J_{z}\right) \dot{\psi}-\dot{h}_{W_{x}}+\omega_{O} h_{W_{z}}+h_{W_{y}} \dot{\psi}+\omega_{o} h_{W_{y}} \varphi \\
& J_{y} \ddot{\theta}=-3 \omega_{O}^{2}\left(J_{x}-J_{z}\right) \theta-\dot{h}_{W y} \\
& J_{z} \ddot{\psi}=-\omega_{O}^{2}\left(J_{y}-J_{x}\right) \psi-\omega_{O}\left(J_{x}-J_{y}+J_{z}\right) \dot{\varphi}-\dot{h}_{W_{z}}-\omega_{o} h_{W_{x}}-h_{W_{y}} \dot{\varphi}+\omega_{o} h_{W_{y}} \dot{\psi}
\end{aligned}
$$

(61) represents the rotational motion of the satellite including gravity gradient torque and reaction wheels. The pitch equation is decoupled from the roll and azimuth equations. Roll and azimuth are coupled through the bias momentum, $h_{w y}$, and the orbit rate term $\left(\mathbf{J}_{\mathrm{x}}-\mathbf{J}_{\mathbf{y}}+\mathbf{J}_{\mathrm{z}}\right) \omega_{\mathrm{o}}$.

\subsubsection{Magnetic Coil Torque}

The equation of the magnetic control torque is given by (24) and may be expressed as

$$
{ }^{B} T_{m}=S\left({ }^{B} m\right)^{o} B=\left[\begin{array}{c}
2 m_{y} \sin \lambda_{m} \\
m_{z} \cos \lambda_{m}-2 m_{x} \sin \lambda_{m} \\
-m_{y} \cos \lambda_{m}
\end{array}\right]
$$

where ${ }^{\circ} \mathrm{B}$ is given by (31) and

$$
S\left({ }^{B} m\right)=\left[\begin{array}{ccc}
0 & -m_{z} & m_{y} \\
m_{z} & 0 & -m_{x} \\
-m_{y} & m_{x} & 0
\end{array}\right]
$$

The rotational motion of the satellite including gravity gradient torque and magnetic coil torque written in component form becomes

$$
\begin{aligned}
& J_{x} \ddot{\varphi}=-4 \omega_{O}^{2}\left(J_{y}-J_{z}\right) \varphi+\omega_{O}\left(J_{x}-J_{y}+J_{z}\right) \dot{\psi}+2 B_{o} m_{y} \sin \lambda_{m} \\
& J_{y} \ddot{\theta}=-3 \omega_{O}^{2}\left(J_{x}-J_{z}\right) \theta-2 B_{O} m_{x} \sin \lambda_{m}+B_{O} m_{z} \cos \lambda_{m} \\
& J_{z} \ddot{\psi}=-\omega_{O}^{2}\left(J_{y}-J_{x}\right) \psi-\omega_{O}\left(J_{x}-J_{y}+J_{z}\right) \dot{\varphi}-B_{O} m_{y} \cos \lambda_{m}
\end{aligned}
$$

(63) can be represented as a state-space model

$$
\left[\begin{array}{c}
\dot{x}_{1} \\
\dot{x}_{2} \\
\dot{x}_{3} \\
\dot{x}_{4} \\
\dot{x}_{5} \\
\dot{x}_{6}
\end{array}\right]
$$

$$
=\left|\begin{array}{cccccc}
0 & 1 & 0 & 0 & 0 & 0 \\
-\frac{4 \omega_{O}^{2}\left(J_{y}-J_{z}\right)}{J_{x}} & 0 & 0 & 0 & 0 & \frac{\omega_{O}\left(J_{x}-J_{y}+J_{z}\right)}{J_{x}} \\
0 & 0 & 0 & 1 & 0 & 0 \\
0 & 0 & -\frac{3 \omega_{O}^{2}\left(J_{x}-J_{z}\right)}{J_{y}} & 0 & 0 & 0 \\
0 & 0 & 0 & 0 & 0 & 1 \\
0 & -\frac{\omega_{O}\left(J_{x}-J_{y}+J_{z}\right)}{J_{x}} & 0 & 0-\frac{\omega_{O}^{2}\left(J_{y}-J_{x}\right)}{J_{z}} & 0
\end{array}\right|\left|\begin{array}{l}
x_{1} \\
x_{2} \\
x_{3} \\
x_{4} \\
x_{5} \\
x_{6}
\end{array}\right|
$$




$$
+B_{O}\left|\begin{array}{ccc}
0 & 0 & 0 \\
0 & \frac{2 \sin \lambda_{m}(t)}{J_{x}} & 0 \\
0 & 0 & 0 \\
-\frac{2 \sin \lambda_{m}(t)}{J_{y}} & 0 & \frac{\cos \lambda_{m}(t)}{J_{y}} \\
0 & 0 & 0 \\
0 & -\frac{\cos \lambda_{m}(t)}{J_{z}} & 0
\end{array}\right|\left[\begin{array}{l}
m_{x} \\
m_{y} \\
m_{z}
\end{array}\right]
$$

The states are given as

$$
x=\left\lfloor\begin{array}{llllll}
\varphi & \dot{\varphi} & \theta & \dot{\theta} & \psi & \dot{\psi}
\end{array}\right\rfloor
$$

\subsubsection{Combine Magnetic Coils and Reaction Wheels}

The magnetic coil torque equation (62) may be superposed into (61) to yield the rotational motion of the satellite controlled from both magnetic coils and reaction wheels.

$$
\begin{aligned}
J_{\lambda} \ddot{\varphi}= & -4 \omega_{o}^{2}\left(J_{y}-J_{z}\right) \varphi+\omega_{O}\left(J_{x}-J_{y}+J_{z}\right) \dot{\psi}-\dot{h}_{W_{x}}+\omega_{o} h_{W_{z}}+h_{W_{y}} \dot{\psi}+\omega_{O} h_{W_{y}} \varphi \\
& +2 B_{O} m_{y} \sin \lambda_{m} \\
J_{y} \ddot{0}= & -3 \omega_{o}^{2}\left(J_{x}-J_{z}\right) \theta-\dot{h}_{W_{y}}-2 B_{o} m_{x} \sin \lambda_{m}+B_{O} m_{z} \cos \lambda_{m} \\
J_{z} \ddot{\psi}= & -\omega_{O}^{2}\left(J_{y}-J_{x}\right) \psi-\omega_{O}\left(J_{x}-J_{y}+J_{z}\right) \dot{\varphi}-\dot{h}_{W_{z}}-\omega_{O} h_{W_{x}}-h_{W y} \dot{\varphi}+\omega_{O} h_{W y} \psi
\end{aligned}
$$

\section{Control and Simulations}

The stability and pointing requirements of the three axes of the satellite will depend on the scientific mission of the satellite. The goal of the following simulations is to indicate which stability and pointing accuracies may be achieved using gravity gradient stabilisation combined with magnetic torquing and/or reaction wheel torquing. The gravitational torque stabilizes the satellite, but resulting low damping requires

Table 1. Applied satellite data

Satellite height:

Moment of inertia about principal axes (boom deployed):

Satellite orbit:

Gravity boom length:

Tip mass:

Magnettorquers:

Reaction wheels:

\section{$1200 \mathrm{~km}$}

$\mathrm{J}_{\mathrm{x}}=178 \mathrm{kgm}^{2}, \mathrm{~J}_{\mathrm{y}}=181 \mathrm{kgm}^{2}, \mathrm{~J}_{\mathrm{z}}=4.3 \mathrm{kgm}^{2}$

Polar orbit

$6 \mathrm{~m}$

$2 \mathrm{~kg}$

3 perpendicular magnetic coils. Each coil gives a magnetic moment of $8 \mathrm{Am}^{2}$

The wheels support torques from 0.01-1 Nm 
additional control torques, see section 4.1. Table 1 lists the applied nominal satellite data.

Three-axis attitude control requires attitude angles and possibly also attitude angular rate information. This paper assumes that angular sensors are chosen such that sufficient accurate roll, pitch and azimuth angle information can be provided. Possible sensors are listed in section 2. Attitude angular rate information, if needed, can be estimated using a Kalman filter, but for simplicity this paper assumes all angular rates to be measurable.

The movements of the satellite are large shortly after release from the space vehicle. This paper assumes that the control system successfully has performed a detumbling operation, and concentrates on the attitude accuracy that can be achieved during small anglc deviations, i.e., in normal space flight. Angular deviation will occur, because the satellite is constantly exposed to disturbance torques (Narheim et al. 1994), owing to

- Magnetic torques from electrical circuits on the satellite

- Solar pressure

- Thermal deformation of the gravity boom

- Atmospheric drag (assumed to be small)

The disturbance torque will try to swing the satellite away from its nominal attitude angles and, hence, a control system is required. The dynamic equations of the satellite are non-linear, coupled and have multiple inputs and outputs. Non-linear systems may require non-linear control. If, however, the simulations are limited to small angle deviations, i.e. after the detumbling phase has ended, the linearized mathematical models presented in chapter 3 may successfully be applied. These linear models also allow linear control strategies. Reaction wheel control is relatively easy to perform. Magnetic torquing gives a discontinuous input matrix and requires a time-varying control. The satellite is released straight above equator, i.e., $\lambda_{\mathrm{m}}=0^{\circ}$, in all simulations.

\subsection{Gravity Gradient Stabilisation-Only}

The deployed gravity boom together with the tip mass should be sufficient to stabilise the satellite. The mathematical model given in $(51,52,53)$ is modelled in MATLAB and simulated using initial conditions imposing the Euler angles. Fig. 4 shows the uncontrolled Euler angles $\varphi, 0, \psi$ against time, the initial conditions are given as $[\varphi \theta \psi]=\left[10^{\circ} 10^{\circ} 10^{\circ}\right]$. Roll angle $=$ solid line, pitch angle $=$ dotted line and azimuth angle $=$ dashed line. One orbit is $6545 \mathrm{sec}$.

The gravitational torque stabilises the satellite, but the damping is very low and each axis oscillates with an amplitude equal the initial imposed amplitude. Additional damping, i.e., additional control torques, are required to remove the oscillations.

\subsection{Gravity Gradient Stabilisation \& Magnetic Coils}

The magnetic coils interact with the magnetic field of the earth and produce a satellite torque. The maximum magnetic dipole moment of the coils is set to $8 \mathrm{Am}^{2}$. It is not straightforward to obtain a good controller using magnetic coils. The process is time-varying, multivariable, coupled and the control action is non-linear. The control torque can be generated perpendicular to the magnetic field vector only, and the roll axis become non-controllable over equator and the azimuth axis become non-controllable over the poles. This can be seen from the B-matrix in (64), where the latitude angle 


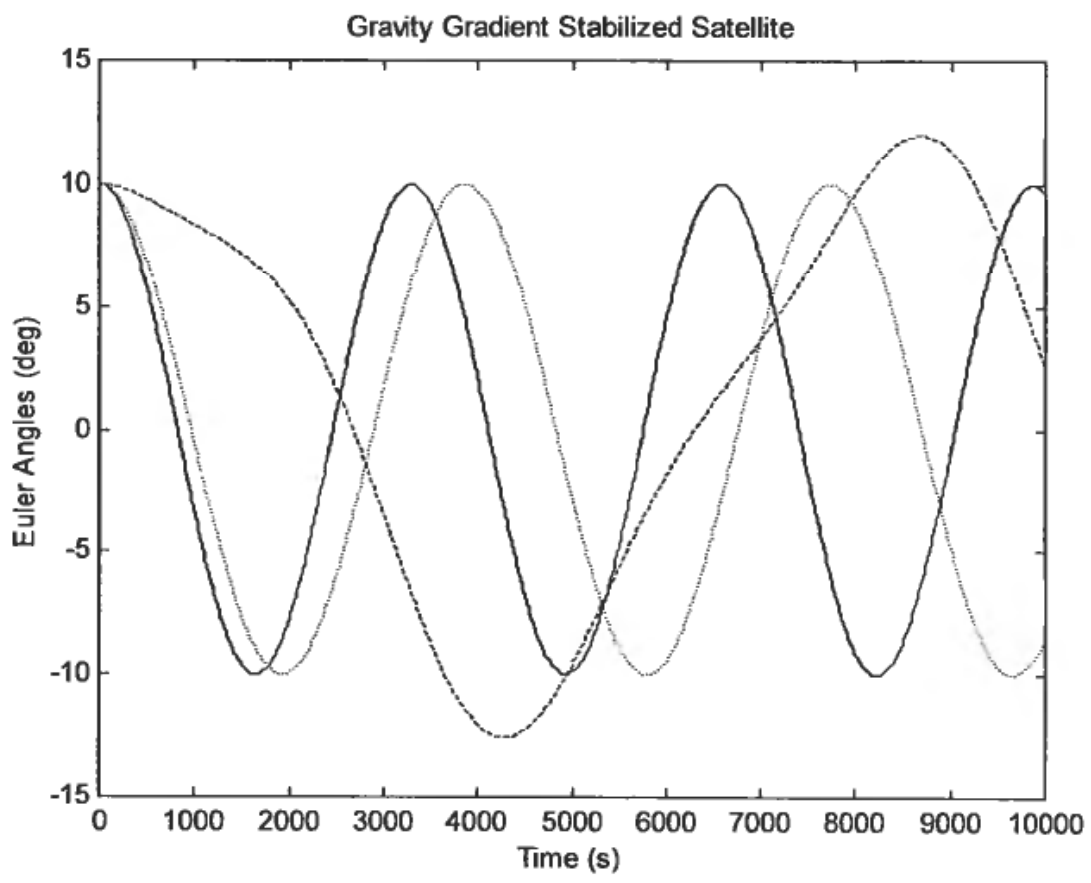

Figure 4. Euler angles against time for a gravity gradient stabilised satellite, exposed to initial angular conditions. Roll angle (-), pitch angle (...) and azimuth angle (--).

with respect to the geomagnetic equatorial plane, $\lambda_{m}$, becomes zero above equator and $90^{\circ}$ above the poles.

The simplest approach to multivariable design is to ignore its multivariable nature. A SISO (single input single output) controller is designed for one pair of input and output variables. When this design has been successfully completed, another SISO controller is designed for a second pair of variables, and so on. This approach was tried, but does not succeed in stabilising all three axes to acceptable values.

As indicated in section 1, several papers describe magnetic torquing using LQ control. An LQ controller calculates the optimal gain G (optimal in the case of linear systems) such that the state-feedback law

$$
u=-G x
$$

minimises the quadratic cost function

$$
J=\int_{0}^{\infty}\left(x^{T}(t) Q x(t)+u^{T}(t) R u(t)\right) d t
$$

$\mathrm{G}$ is derived from

$$
G=R^{-1} B^{T} S
$$

where $S$ is the solution of the Riccati equation.

A LQ controller requires feedback from all states and its derivatives, which means that both the Euler angles and the time derivatives of the Euler angles should be available. The time derivatives of the Euler angles are not measured in the satellite, but these signals can be estimated using a Kalman filter. A Kalman filter may also reduce 


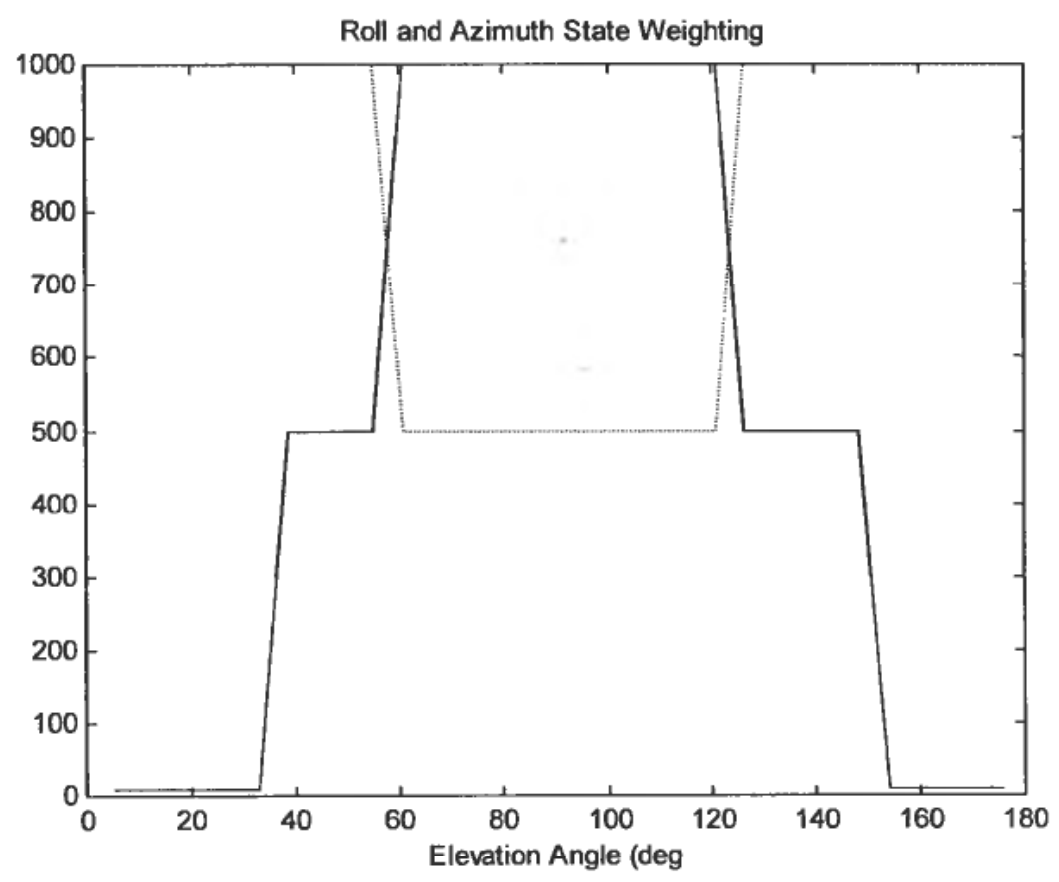

Figure 5. State weight matrix element $\mathrm{q}_{1}(-)$ and $\mathrm{q}_{3}(\ldots)$ against latitude angle.

process and measurement noise in both $\varphi, 0, \psi$ and $\dot{\varphi} \dot{\theta} \dot{\psi}$. A Kalman filter is straight forward to implement, and the simulations in this paper ignore the Kalman filter and simplify by taking all states directly from the process model. The simulation results will to a small extent be influenced by the Kalman filter, supposing sufficient accurate sensors and a properly designed filter. Since the system is time varying, constant state and input weight matrices will be difficult to use. Instead, the latitude angle is frozen at different angles and a LQ controller is calculated at each angle. The state weight matrix is chosen as: $\mathrm{Q}=\operatorname{diag}\left(\mathrm{q}_{1} 1 \mathrm{q}_{2} 1 \mathrm{q}_{3} 1\right)$.

It was found that the angular weights influence the system more than the angular rate weights. Hence, the angular rate weights were set to one, and the angular weights $\mathrm{q}_{1}, \mathrm{q}_{2}, \mathrm{q}_{3}$ were varied according to the latitude angle. The pitch axis is controllable for all latitude angles and not coupled, and $\mathrm{q}_{2}$ was set to a constant value. A guide for choosing a proper state weight matrix $(\mathrm{Q})$ and an input weight matrix $(\mathrm{R})$ can be found in Balcen et al. (1988). Combining these advices with a cut and try approach results in the time-varying $q_{1}$ and $q_{3}$ shown in Fig. 5. The latitude angle, varying from $0-180^{\circ}$, is shown along the $\mathrm{x}$-axis. The values of $\mathrm{q}_{1}$ and $\mathrm{q}_{3}$ are shown along the $\mathrm{y}$-axis. The solid line represents $\mathrm{q}_{1}$ and the dotted line represents $\mathrm{q}_{3}, \mathrm{q}_{2}$ is set to 1000 . The input weight matrix is chosen as: $R=\operatorname{diag}(110001000)$.

The time-varying state weight elements shown in Fig. 5 combined with the constant $\mathrm{q}_{2}$ and the given $\mathrm{R}$ matrix were applied in a simulation and the results are shown in Fig. 6. Fig. 6 a shows the Euler angles $\varphi, \theta, \psi$ against time, the initial conditions are given as $[\varphi \theta \psi]=\left[10^{\circ} 10^{\circ} 10^{\circ}\right],{ }^{\mathrm{B}} \omega_{\mathrm{B} / \mathrm{O}}=0$. The pitch axis is accurately controlled and also the azimuth angle is satisfactorily controlled. The roll axis is weakly damped. Obtaining accurate control in both roll and azimuth turned out to be difficult. Soglo 1994 indicates that changing the moments of inertia of the satellite may improve the stability. Fig. $6 \mathrm{~b}$ 

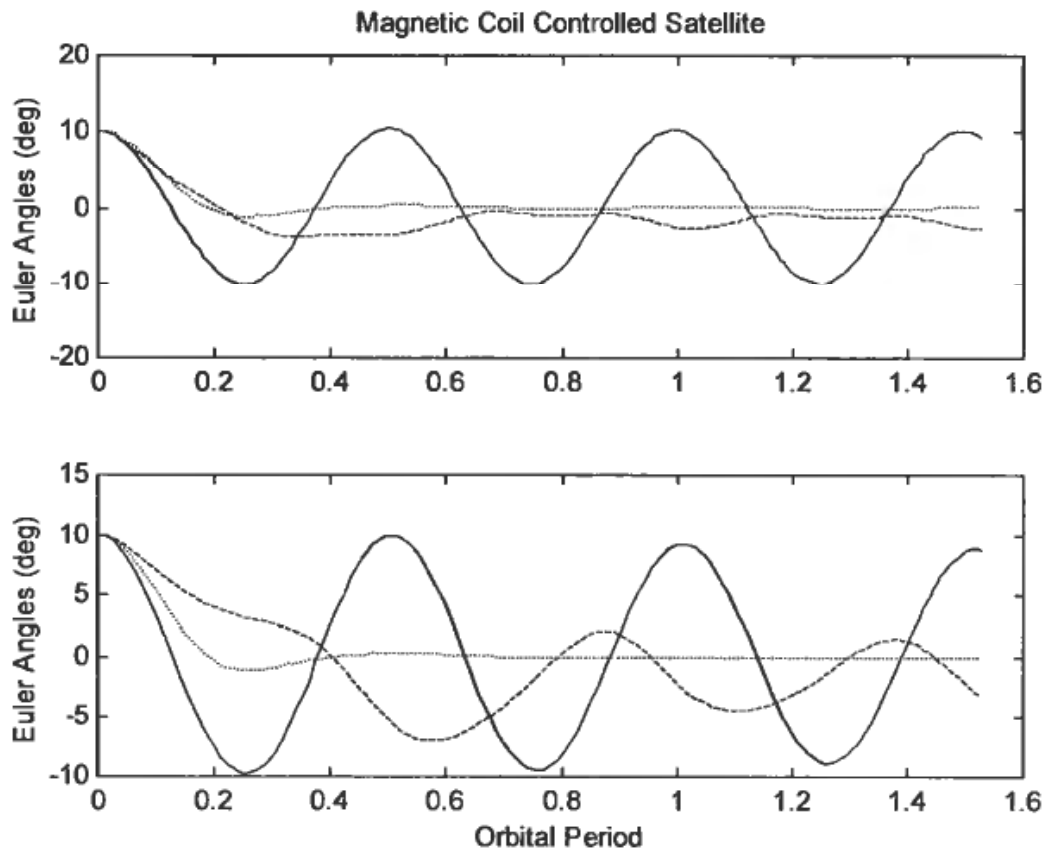

Figure 6a,b. Euler angles against time for a gravity gradient stabilised and magnetic coil controlled satellite, exposed to initial conditions. Roll angle (-), pitch angle (...) and azimuth angle (--).

repeats the simulation performed in Fig. $6 \mathrm{a}$, but $\mathrm{J}_{\mathrm{z}}$ has been changed to $10 \mathrm{kgm}^{2}$. The roll damping is slightly improved at the cost of slightly less damping in azimuth.

The above LQ control can be performed more elegantly by solving the time-varying Riccati equation backwards in time, i.e., calculating the optimal gain at predetermined latitude angles in a more automatic way. Fig. 7a, b, c shows the Euler angles $\varphi, \theta, \psi$ against time, using a LQ controller based on a backward Riccati solution, the initial conditions are given as $[\varphi 0 \psi]=\left[10^{\circ} 10^{\circ} 10^{\circ}\right],{ }^{\mathrm{B}} \omega_{\mathrm{B} / \mathrm{O}}=0$. The state and input weight matrices, using the backward calculation, are chosen as: $Q=\operatorname{diag}(10001000010000)$, $\mathrm{R}=\operatorname{diag}\left(\begin{array}{lll}1 & 1 & 1\end{array}\right)$.

The pitch axis is again accurately controlled, also the damping in azimuth is acceptable, but the roll axis is, as shown in Fig. 7a, too lightly damped. Increasing the coil moments hardly influences the result. Neither are significant improvements obtained by completely removing the coil moment limits. Changing the moment of inertias, however, allow an improved roll stability. Fig. $7 \mathrm{~b}$ shows a simulation where $J_{z}$ has been increased from $4.3 \mathrm{kgm}^{2}$ to $14.3 \mathrm{kgm}^{2}$. Increasing $J_{z}$ improves the damping of the $x$-axis. Better results are obtained by using even larger $J_{z}$ values, as shown in Fig. $7 \mathrm{c}$, where $\mathrm{J}_{\mathrm{z}}=34.3 \mathrm{kgm}^{2}$. Nominally, the satellite has a moment of inertia ratio of $178 / 4.3=41.4$ between the $x$-axis and the $z$-axis. The same magnetic coil simultaneously controls the $\mathrm{x}$-axis and the $\mathrm{z}$-axis. The LQ controller is not able to effectively handle this large moment of inertia ratio using the same actuator, and lowering the ratio makes the LQ controller more effective. Fig. 7a and b show a slowly decreasing roll angle and to see if this damping is real, the simulation shown in Fig. $7 \mathrm{~b}$ is repeated using a simulation time of 8 orbits. The extended Fig. 7b simulation shows a roll angle of 

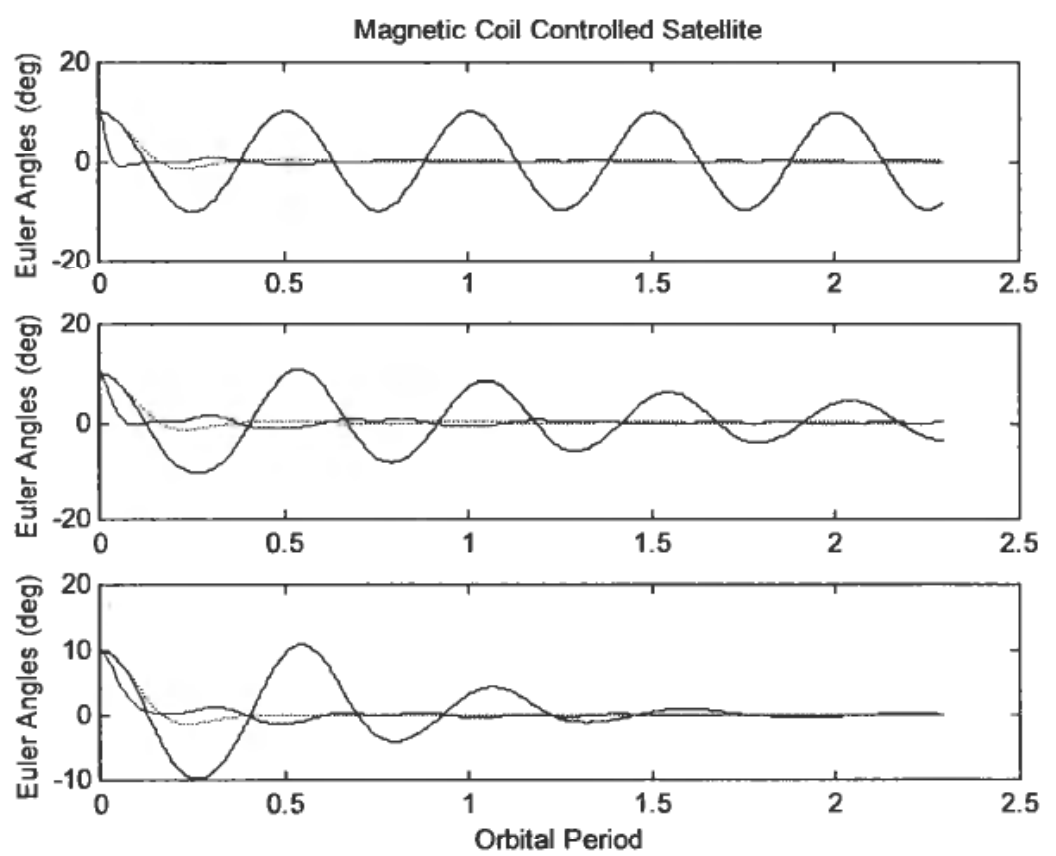

Figure 7a,b,c. Euler angles against time for a gravity gradient and magnetic coil controlled satellite, exposed to initial angular conditions. Roll angle $(-)$, pitch angle (...) and azimuth angle (--).

$\pm 0.5^{\circ}$ after 7 orbits and the angle is still decreasing. The damping is real and, providing no disturbances, the roll angle goes to a small value.

\subsection{Reaction Wheels \& Gravity Stabilisation}

An alternative way of controlling the satellite is to apply three reaction wheels, acting around each of the three body-axes. (61) is modelled in MATLAB and a LQ controller is developed to perform the attitude control. The input matrix is time-invariant and the Riccati equation can be solved the normal way, i.e., forward in time. Fig. 8a, b, c shows the Euler angles against time using initial conditions $[\varphi 0 \psi]=\left[10^{\circ} 10^{\circ} 10^{\circ}\right],{ }^{\mathrm{B}} \omega_{\mathrm{B} / \mathrm{O}}=0$. Fig. 8a shows the result using reaction wheels limited to a torque of $1 \mathrm{Nm}$. In Fig. $8 \mathrm{~b}$ the torques are limited to $0.1 \mathrm{Nm}$ and in Fig. $8 \mathrm{c}$ to $0.01 \mathrm{Nm}$. Fig. 8a shows that the reaction wheels supporting $1 \mathrm{Nm}$ very quickly stabilise the satellite in azimuth. This fast response is due to the low moment of inertia in azimuth. Roll and pitch stabilisation is slower, but still very quick compared to the magnetic coils. Decreasing the available torque to $0.1 \mathrm{Nm}$, shown in Fig. $8 \mathrm{~b}$, made roll and pitch slower and slightly less stable, but still very quick. Even a maximum torque of $0.01 \mathrm{Nm}$, shown in Fig. 8c, gives a very tight and fast control, all compared to the magnetic coil control.

It is possible to further decrease the size of the reaction wheels, but at the cost of slower response, and possibly also less damping. A mathematical model of the reaction wheels, including a realistic friction and stiction model, is needed to establish a more precise lower limit for the wheel torques. Fig. 8a and $\mathrm{b}$ use the state and input weight matrices: $Q=\operatorname{diag}(0.110000 .11000101000), R=\operatorname{diag}(0.10 .10 .1)$. Fig. $8 c$ 

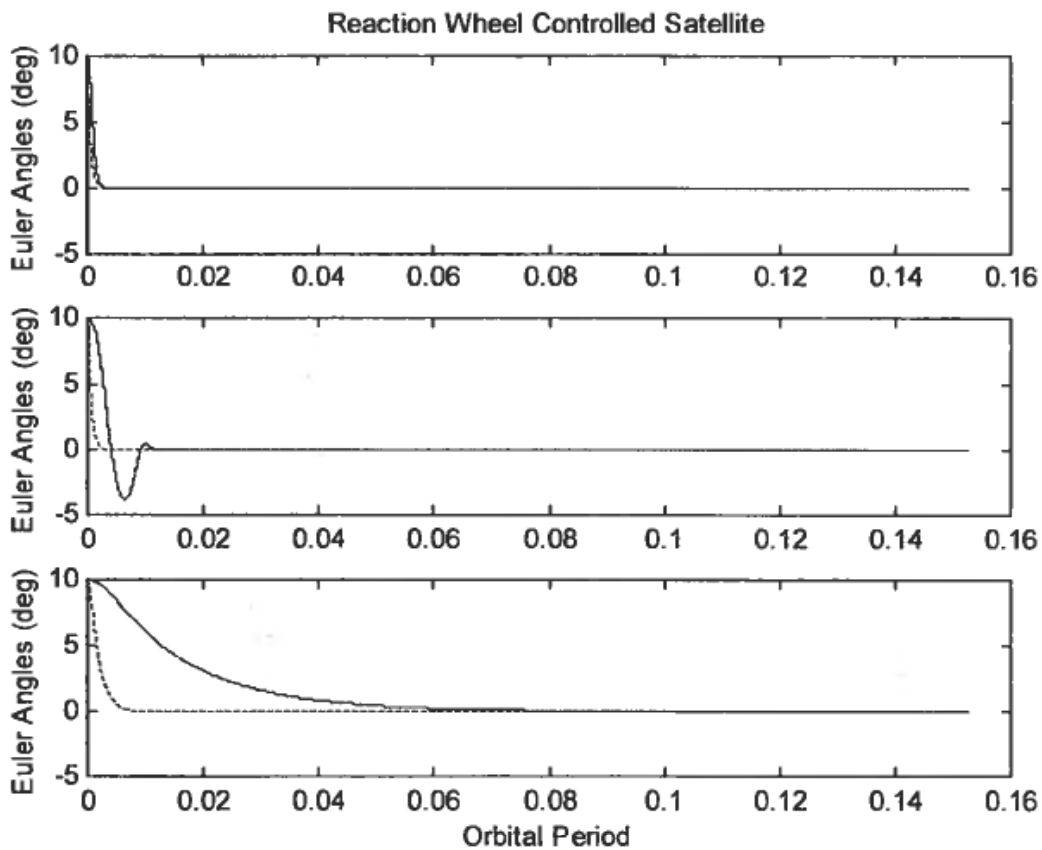

Figure 8a,b,c. Euler angles against time for a gravity gradient stabilised and reaction wheel controlled satellite, exposed to initial angular conditions. Roll angle (-), pitch angle (...) and azimuth angle (--).

uses the state and input weight matrices: $Q=\operatorname{diag}(100100010010001001000)$, $\mathrm{R}=\operatorname{diag}(0 \cdot 10 \cdot 10 \cdot 1)$.

\subsection{Combine Magnetic Coils and Reaction Wheels}

Section 4.2 shows that it was difficult to achieve accurate attitude controls in both roll and azimuth using magnetic coils and gravitational stabilisation. In this section a reaction wheel is mounted to control the satellite in roll. Pitch and azimuth are controlled from magnetic coils. The corresponding mathematical model is shown in (69)

$$
\begin{aligned}
J_{x} \ddot{\varphi}= & -4 \omega_{O}^{2}\left(J_{y}-J_{z}\right) \varphi+\omega_{O}\left(J_{x}-J_{y}+J_{z}\right) \dot{\psi}-\dot{h}_{W x} \\
J_{y} \ddot{\theta}= & -3 \omega_{O}^{2}\left(J_{x}-J_{z}\right) \theta-\dot{h}_{W y}-2 B_{O} m_{x} \sin \lambda_{m}+B_{O} m_{z} \cos \lambda_{m} \\
J_{z} \ddot{\psi}= & -\omega_{O}^{2}\left(J_{y}-J_{x}\right) \psi-\omega_{O}\left(J_{x}-J_{y}+J_{z}\right) \dot{\varphi}-\dot{h}_{W_{z}}-\omega_{O} h_{W_{x}}-h_{W y} \dot{\varphi}+\omega_{O} h_{W_{y}} \psi \\
& -B_{O} m_{y} \cos \lambda_{m}
\end{aligned}
$$

Fig. 9a, b, c show the simulation results obtained using the initial conditions $[\varphi \theta \psi]=\left[10^{\circ} 10^{\circ} 10^{\circ}\right],{ }^{\mathrm{B}} \omega_{\mathrm{B} / \mathrm{O}}=0$. Maximum torque of the roll wheel is set to $0.1 \mathrm{Nm}$, the magnetic coils in pitch and roll are limited to $8 \mathrm{Am}^{2}$. Different state and input matrices are tried in $\mathrm{a}, \mathrm{b}$ and $\mathrm{c}$.

\section{Conclusion}

This article develops a linearized mathematical model of a proposed Norwegian 

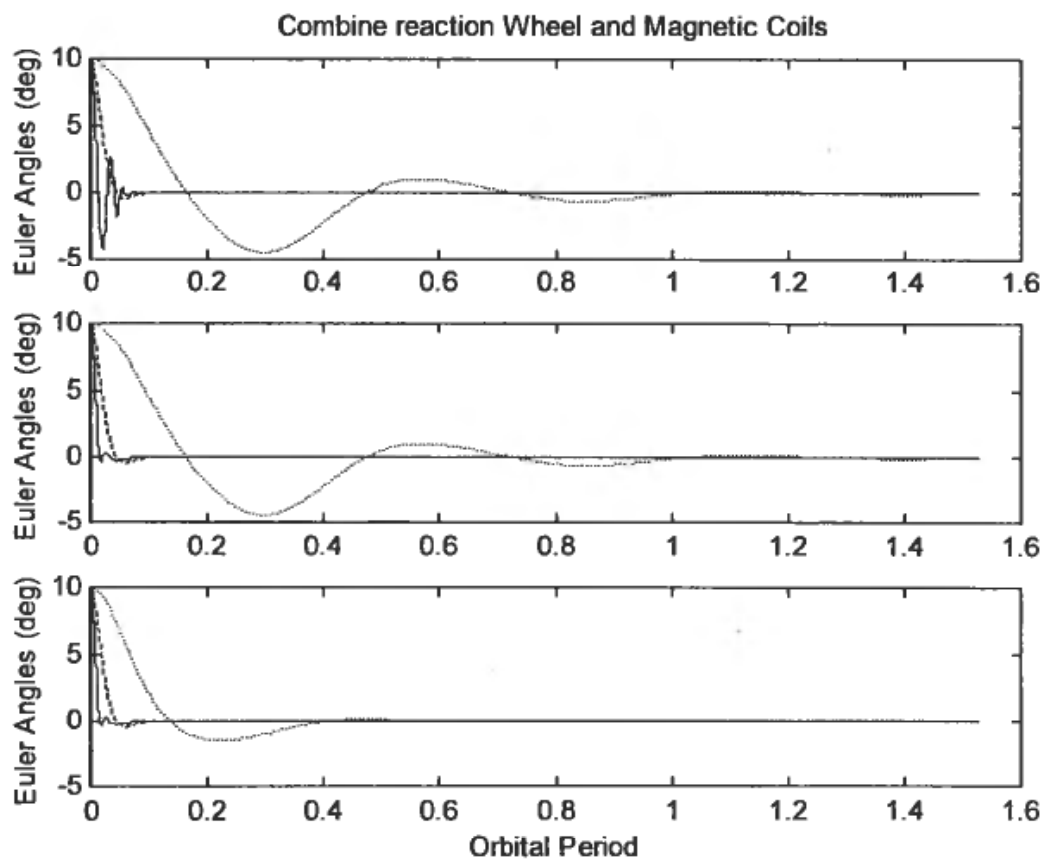

Figure 9. Euler angles against time for a gravity gradient stabilised and coil/wheel controlled satellite, exposed to initial angular conditions. Roll angle (-), pitch angle (...) and azimuth angle (--).

small satellite. The satellite is intended to fly in a low orbit and perform scientific measurements. The satellite is to be gravity gradient stabilised.

Simulations have shown that gravity gradient stabilisation alone does not give the stability and pointing accuracy that many scientific measurements require, hence, additional actuators should be added.

Magnetic coils are attractive actuators for small cheap satellites. Different controllers are considered in order to obtain the best possible three-axis control. LQ controllers gave best results, but the simulations also reveal that accurate three-axis control is difficult to achieve using magnetic coils and the proposed moments of inertias. A time-varying magnetic field, which causes roll and azimuth to become non-controllable in parts of the orbits, is to blame. However, changing the moment of inertia improves the damping and simulations indicate a pointing accuracy down to $3-7^{\circ}$ in roll and azimuth and $1-4^{\circ}$ in pitch, depending on the chosen moment of inertias and also the size of the disturbances.

Replacing the magnetic coils with reaction wheels gave very accurate attitude control. It is expected that an accuracy, depending on the size of the wheels, down to less than $1^{\circ}$ in all three axes can be achieved.

A cheaper way of obtaining a relatively accurate attitude control is to replace the magnetic coil that controls roll with a reaction wheel, i.e., pitch and azimuth are controlled from magnetic coils and roll from a wheel. This actuator combination is expected to give an accuracy of less than $1^{\circ}$ in roll and approximately $1-4^{\circ}$ in pitch and azimuth. 


\section{REFERENCES}

BaK, T., Wisniewski, R. and Blanke, M. (1996). Autonomous Attitude Determination and Control System for the Ørsted Satellite. In proc.: IEEE Aerospace Application Conference.

Balchen, J. G. and Mumme, K. I. (1988). Process Control: Structures and Applications. Van Nostrand Reinhold.

Bryson, A. E. (1994). Control of Spacecraft and Aircraft. Princeton University Press, New Jersey.

Cavallo, A., De Maria, G., Ferrara, F. and Nistri, P. (1993). A Sliding Manifold Approach to Satellite Attitude Control. In proc.: 12th World Congress IFAC, Sydney.

EGELAND, O. (1993). Robotdynamikk, Lecture notes. NTNU.

Fossen, T. I. (1994). Guidance and Control of Ocean Vehicles, John Wiley \& Sons, Inc.

HodgarT, M. and ONG, W. (1994). Gravity Gradient and Magnettorquing Attitude Control for Low Orbit Satellites. 2nd ESA Int. Conf. Spacecraft Guidance, Navigation and Control Systems, ESTEC.

HugHES, P. C. (1986). Spacecraft dynamics, John Wiley \& Sons, Inc.

KANE, T. K., LikINS, P. W. and LeVInSON, D. A. (1983). Spacecraft Dynamics, McGraw-Hill, Inc.

KaPlan, M. H. (1976). Modern Spacecraft Dynamics and Control. John Wiley, New York.

MARTEl, F., PARIMAL, K. P. and PSIAKI, M. (1988). Active Magnetic Control System for Gravity Gradient Stabilized Spacecraft. In proc.: Annual AIAA/Utah State University Conference on Small Satellites.

MuSSER, K. L. and WARD, L.E. (1989). Autonomous Spacecraft Attitude Control using Magnetic Torquing Only. In proc.: Flight Mechanics Estimation Theory Symposium, NASA.

NARHEIm, B. T. and SvenES, K. R. (1994). Norwegian Ionospheric Small Satellite Experiment (NISSE). Phase-A Study Report.

LEWIS, F. L. (1986). Optimal Control. John Wiley \& Sons.

SKULLESTAD, A. (1993). Lecture notes in robotteknikk. HIBU.

SKULLESTAD, Å. (1995). Identification of vibration parameters in a space structure. PhD Thesis, University of Oslo.

SOGLO, P. K. (1994). 3-aksestyring av gravitasjonsstabilisert satellitt ved bruk av magnetspoler, Hovedoppgave NTNU.

STICKLER, A. C. (1974). Elementary Magnetic Attitude Control System. In proc.: AIAA Mechanics and Control of Flight Conference.

WIESEL, W. E. (1989). Spaceflight Dynamics, McGraw-Hill, Inc.

WERTZ, J. R. (1978). Spacecraft Attitude Determination and Control, Kluwer Academic Publishers.

WiSNIEWSKI, R. (1996). Satellite Attitude Control Using Only Electromagnetic Acutation. PhD Thesis, University of Aalborg. 